\title{
Pérdida de seguimiento y factores asociados en pacientes inscritos en el programa de HIV/sida del Hospital Universitario San Ignacio, Colombia, 2012-2013
}

\author{
José Roberto Támara-Ramírez ${ }^{1}$, Carlos Arturo Álvarez², Jesús Rodríguez ${ }^{3}$ \\ Grupo de Investigación en Enfermedades Infecciosas, Hospital Universitario San Ignacio, Pontificia Universidad \\ Javeriana, Bogotá, D.C., Colombia \\ 2 Clínica Universitaria Colombia, Colsanitas, S.A., Bogotá, D.C., Colombia \\ 3 Centro de Proyectos para el Desarrollo (CENDEX), Pontificia Universidad Javeriana, Bogotá, D.C., Colombia
}

Introducción. El seguimiento clínico regular a largo plazo, es un componente importante en la atención de los pacientes con HIV.

Objetivo. Describir las razones de la pérdida de seguimiento de los pacientes inscritos en el programa de HIV/sida de un hospital universitario.

Materiales y métodos. Se hizo un estudio de casos y controles anidado en una cohorte retrospectiva, entre el $1^{\circ}$ de enero de 2012 y el 31 de julio de 2013.

Resultados. Se seleccionaron 45 pacientes; la tasa de densidad de incidencia de los pacientes que se perdieron en el seguimiento fue de 17,7 por 100 pacientes por año. En el análisis bivariado, el desempleo $(p=0,000)$, el consumo de alcohol $(p=0,004)$, el tiempo de la evolución de la enfermedad en años $(p=0,032)$, el sexo $(p=0,027)$, y la edad promedio de 34 años $(p=0,000)$ fueron estadísticamente significativos en relación con la pérdida de seguimiento. Al hacer el ajuste por regresión logística para la probabilidad de pérdida de seguimiento, se evidenció que las variables de edad promedio de 34 años $\left(\mathrm{p}=0,019 ; \mathrm{IC} \mathrm{C}_{95 \%} 0,871-0,976\right)$, sexo femenino $\left(\mathrm{p}=0,017 ; \mathrm{IC} \mathrm{C}_{95 \%} 1,903-31,83\right)$, consumo de alcohol $(\mathrm{p}=0,028$; $\left.\mathrm{IC}_{95 \%} 0,040-0,830\right)$ y desempleo ( $\left.\mathrm{p}=0,001 ; \mathrm{IC}_{95 \%} 4,696-464,692\right)$ tuvieron significación estadística.

Conclusiones. Es necesario que los programas de HIV/sida establezcan sistemas de seguimiento y rastreo, con el fin de establecer estrategias para mejorar la retención de los pacientes y, por ende, su calidad de vida a largo plazo.

Palabras clave: infecciones por HIV, síndrome de inmunodeficiencia adquirida/terapia, pérdida de seguimiento, factores de riesgo, incidencia.

doi: http://dx.doi.org/10.7705/biomedica.v36i2.2939

\section{Loss of follow-up and associated factors in patients enrolled in the HIV/AIDS program of the Hospital Universitario San Ignacio, Colombia, 2012-2013}

Introduction: Regular long-term clinical follow-up is an important component of HIV care.

Objective: To describe the reasons for follow-up loss among patients enrolled in the HIV/AIDS program of a university hospital.

Materials and methods: A nested case-control study was carried out on a retrospective cohort between January $1^{\text {st }}, 2012$ and July $31^{\text {st }}, 2013$.

Results: A group of 45 patients was selected; the incidence density rate of patients lost to follow-up was 17.7 per 100 patient/years. The following variables were significantly linked to follow-up loss in the bivariate analysis: Unemployment $(p=0.000)$; alcohol consumption $(p=0.004)$; number of years of evolution of the disease $(p=0.032)$; gender $(p=0.027)$, and mean age of 34 years $(p=0.000)$. When logistic regression was adjusted for the probability of follow-up loss the significant variables were: Mean age of 34 years ( $p=0.019,95 \% \mathrm{Cl}: 0.871-0.976)$; female ( $p=0.017,95 \% \mathrm{Cl}: 1.903-31.83)$; alcohol consumption ( $p=0.028,95 \% \mathrm{Cl}: 0.040-0.830$ ), and unemployment ( $\mathrm{p}=0.001,95 \% \mathrm{Cl}: 4.696-464.692)$. Conclusions: HIV/AIDS programs need to establish follow-up systems and means to trace any losses in order to establish strategies to improve patient retention and, thus, their long-term quality of life.

Key words: HIV infections, acquired immunodeficiency syndrome/therapy, lost to follow-up, risk factors, incidence.

doi: http://dx.doi.org/10.7705/biomedica.v36i2.2939

\section{Contribución de los autores:}

José Roberto Támara-Ramírez: concepción, diseño, base de datos, entrevista, recolección e interpretación de los datos Jesús Rodríguez y Carlos Arturo Álvarez: ajuste del diseño y análisis estadístico

Todos los autores participaron en la redacción y revisión del artículo. 
La disponibilidad del tratamiento antirretroviral de gran eficacia ha transformado el pronóstico del paciente infectado con el virus de inmunodeficiencia humana (HIV) en los últimos años $(1,2)$, lo cual ha significado una reducción acentuada en la morbilidad y la mortalidad relacionadas con la infección (3). En el manejo a largo plazo del paciente con HIV, han surgido retos adicionales como la toxicidad de los medicamentos $(4,5)$, el fracaso del tratamiento debido a un cumplimiento inadecuado $(6,7)$, la resistencia a los medicamentos antirretrovirales (8) con el consecuente cambio en el esquema de tratamiento, y un incremento en la morbilidad y la mortalidad no asociadas al HIV $(9,10)$. Los pacientes con HIV requieren un seguimiento clínico prolongado para vigilar la progresión de la enfermedad y determinar el momento oportuno para iniciar el tratamiento y para evaluar la respuesta terapéutica, el cumplimiento del tratamiento antirretroviral y los efectos secundarios, y para abordar los aspectos de salud sexual y las necesidades de prevención del HIV (11).

En el 2006 se diseñó en Colombia el modelo de gestión programática en HIV/sida, según lo recomendado en la guía de atención formulada en el 2000 por el Ministerio de Salud (Resolución 412), que hoy se denomina "Guía de atención en HIV basada en la evidencia". Los objetivos de dicha guía fueron los de contener la epidemia, detectar tempranamente la infección por HIV, detener su progresión a sida y evitar la aparición de resistencia al tratamiento antirretroviral, y, por último, vigilar y evaluar el tratamiento a largo plazo $(12,13)$.

La pérdida de seguimiento es perjudicial para el asesoramiento y el seguimiento clínico y de laboratorio, lo que trae consecuencias individuales y colectivas, ya que el éxito virológico (supresión de la carga viral del HIV mediante el tratamiento antirretroviral) se relaciona con el aumento de la supervivencia (14) y con la reducción de la transmisión del virus (15), mientras que la pérdida de seguimiento y la consecuente interrupción del tratamiento pueden ser un marcador de progresión

\footnotetext{
Correspondencia:

José Roberto Támara, Grupo de Investigación en Enfermedades Infecciosas, Hospital Universitario San Ignacio, Pontificia Universidad Javeriana, Carrera $7 \mathrm{~N}^{\circ}$ 40-62, Bogotá, D.C., Colombia

Teléfono: (571) 594 6161, extensión 2930

jrtamara2101@gmail.com

Recibido: 02/07/15; aceptado: 02/11/15
}

de la enfermedad o de muerte, que en el plano colectivo se asocia con una mayor probabilidad de transmisión del HIV (16).

La retención a largo plazo de los pacientes con HIV es, por lo tanto, un componente importante de su atención. Además, las altas tasas de pérdida de seguimiento podrían comprometer la eficacia de la atención de estos pacientes, por lo cual es necesario conocer las tasas y las razones de la pérdida de seguimiento en nuestro país, con el fin de desarrollar estrategias para optimizar la retención en los programas de atención de HIV/sida.

\section{Materiales y métodos}

Se hizo un estudio de casos y controles anidado en una cohorte retrospectiva, entre el $1^{\circ}$ de enero de 2012 y el 31 de julio de 2013. Se tomó como muestra la población de pacientes del programa de HIV/sida del Hospital Universitario San Ignacio de Bogotá, y se consideraron como casos todos aquellos que hubieran faltado durante tres o más meses a la consulta y, como controles, a quienes seguían asistiendo a las citas de seguimiento; los participantes se seleccionaron de forma secuencial en la misma cohorte hasta completar dos controles por caso.

La pérdida de seguimiento se definió como la ausencia de un paciente durante tres meses o más a partir de la última cita de control. Se consideraron cortes a los cuatro, cinco y seis meses o más.

Se diseñó un formato de entrevista para indagar sobre los factores asociados a la pérdida de seguimiento en un programa de atención en salud de pacientes con HIV/sida. El cuestionario se ajustó después de cinco entrevistas con pacientes que no participaban en el estudio, con quienes se hizo la prueba piloto.

Los datos del cuestionario se resumieron en medidas de tendencia central y de dispersión para las variables cuantitativas, y en frecuencias absolutas y relativas para las variables cualitativas.

\section{Análisis descriptivo}

Los pacientes definidos como casos de pérdida en el seguimiento, se caracterizaron según las variables sujetas a medición. Se calculó la tasa de densidad de la incidencia del programa de HIV/sida del Hospital Universitario San Ignacio, representada por los nuevos casos de pacientes perdidos en el seguimiento en relación con el número de personas por año durante el tiempo del estudio. 


\section{Análisis comparativo bivariado}

Los pacientes se agruparon en dos grupos: los que se mantenían en el seguimiento y aquellos que no habían continuado el seguimiento. Se identificaron las diferencias entre los grupos con respecto a las variables de edad, sexo, nivel educativo, ocupación, tipo de ocupación, acceso a los servicios de salud, lugar de residencia, modo de contagio, tiempo de evolución de la enfermedad en años, carga viral basal, carga viral posterior a la pérdida de seguimiento, tratamiento antirretroviral, suspensión del tratamiento antirretroviral, falla del tratamiento, tabaquismo, consumo de drogas psicoactivas (drogadicción), consumo de alcohol, alteración del estado de ánimo, atención psicológica, hospitalizaciones, tiempo de pérdida de seguimiento y perdida de seguimiento previo. Para la determinación de las diferencias se utilizaron las pruebas t de Student y ji al cuadrado.

\section{Análisis comparativo multivariado}

Las variables que resultaron asociadas estadísticamente con la pertenencia o no al grupo de pacientes perdidos en el seguimiento al hacer el análisis bivariado, se incluyeron en un modelo multivariado de regresión logística para evaluar la importancia relativa de cada una frente a la probabilidad de pertenecer al grupo de perdidos en el seguimiento, en tanto que se controlaba el efecto de las restantes.

Para evaluar el ajuste del modelo de regresión logística, se utilizó el test de Hosmer y Lemeshow. En todas las pruebas el nivel de significación fue de $5 \%$ para la identificación de las diferencias estadísticamente significativas. Para el procesamiento de los datos se utilizó el paquete estadístico SPSS ${ }^{\circledR}$ (IBM Corp., released 2013. IBM SPSS Statistics for Windows, version 22.0. Armonk, NY: IBM Corp.).

\section{Consideraciones éticas}

Este trabajo contó con el aval del Comité de Ética Médica del Hospital Universitario San Ignacio y se clasificó como de mínimo riesgo según los criterios de la Declaración de Helsinki y de la guía de buenas prácticas clínicas. La participación de los pacientes fue libre y voluntaria, y todos los seleccionados firmaron el consentimiento informado.

\section{Resultados}

El programa de HIV/sida del Hospital Universitario San Ignacio contaba con una población de 1.691 pacientes mayores de 18 años durante el periodo del estudio (19 meses de seguimiento, entre el $1^{\circ}$ de enero de 2012 y el 31 de julio de 2013). De dicho total, $1.004(59,4 \%)$ se mantenían en el seguimiento, $347(20,5 \%)$ habían sido trasladados a otra institución prestadora de salud (IPS), 137 $(8,1 \%)$ asistían a control cada cuatro o seis meses y no recibían tratamiento antirretroviral, y $203(12 \%)$ se habían perdido en el seguimiento (casos). La tasa de densidad de la incidencia de los pacientes perdidos en el seguimiento fue de 17,74 por 100 personas por año (203/1.143,75 pacientes por año).

Se citaron y entrevistaron 45 pacientes del total de los considerados como perdidos en el seguimiento (casos): 38 hombres $(84,44 \%)$ y siete mujeres (15,55 \%). Dos de estos pacientes no se incluyeron en el análisis por no tener la información completa. Del grupo de pacientes que cumplían con el seguimiento, se escogieron al azar 85 que conformaron el grupo de control del estudio. Las características sociodemográficas y de comportamiento de los pacientes perdidos (casos), se describen en el cuadro 1. En el cuadro 2 se señalan las 21 razones expuestas por los 43 pacientes para haberse ausentado del programa de HIV/sida del hospital, las cuales se clasificaron en tres grandes grupos: factores administrativos del aseguramiento, factores ocupacionales y factores psicosociales.

Los hallazgos del análisis de casos y controles se describen en el cuadro 3 , en el cual se resalta que la inclusión en el grupo de casos (perdidos en el seguimiento) fue estadísticamente significativo; siete mujeres (16,3\%) y cuatro hombres $(4,7 \%)$ quedaron incluidos en el grupo de control, es decir, el conformado por los pacientes que continuaban en el seguimiento $(p=0,027)$. En la variable de desempleo hubo 18 pacientes perdidos en el seguimiento $(41,9 \%)$, y ninguno en el grupo de control $(p=0,000)$, y el consumo de alcohol fue la razón más frecuente dada por los integrantes del grupo de casos en comparación con el grupo de control $(p=0,006)$.

En el análisis multivariado (cuadro 4) se seleccionaron cinco variables, en algunas de las cuales se hicieron categorizaciones adicionales para facilitar la interpretación de los resultados. La edad $(p=0,027)$, el empleo o renta y el desempleo $(p=0,000)$, el consumo de alcohol $(p=0,004)$, el tiempo de evolución de la enfermedad en años $(p=0,032)$ y la edad promedio $(p=0,000)$, se asociaron estadísticamente con la pérdida en el seguimiento en el grupo de los casos. Las variables de sexo $(p=0,017)$, 
Cuadro 1. Características sociodemográficas y de comportamiento de los pacientes perdidos en el seguimiento

\begin{tabular}{|c|c|c|c|c|c|}
\hline \multirow[t]{2}{*}{ Variables } & \multicolumn{2}{|c|}{ Hombres } & \multicolumn{2}{|c|}{ Mujeres } & \multirow[t]{2}{*}{$p$} \\
\hline & $\mathbf{n}$ & $\%$ & $\mathbf{n}$ & $\%$ & \\
\hline \multicolumn{6}{|l|}{ Grupos de edad (años) } \\
\hline 20 a 30 & 12 & 33 & 3 & 42,9 & 0,913 \\
\hline 31 a 40 & 15 & 41,7 & 3 & 42,9 & \\
\hline 41 a 50 & 8 & 22,2 & 1 & 14,3 & \\
\hline 51 y más & 1 & 2,8 & 0 & 0 & \\
\hline Escolaridad & & & & & \\
\hline Primaria incompleta & 2 & 5,6 & 0 & 0 & 0,217 \\
\hline Primaria completa & 2 & 5,6 & 1 & 14,3 & \\
\hline Secundaria incompleta & 2 & 5,6 & 0 & 0 & \\
\hline Secundaria completa & 5 & 13,9 & 3 & 42,9 & \\
\hline Técnica & 11 & 30,6 & 3 & 42,9 & \\
\hline Universitaria & 14 & 38,9 & 0 & 0 & \\
\hline Ocupación & & & & & \\
\hline Ocupado & 20 & 55,6 & 4 & 57,1 & 0,938 \\
\hline Desocupado & 16 & 44,4 & 3 & 42,9 & \\
\hline Obrero/empleado & 12 & 33,3 & 4 & 57,1 & \\
\hline Patrón/empleador & 3 & 8,3 & 0 & 0 & \\
\hline Tipo de ocupación & & & & & \\
\hline Trabajador independiente & 5 & 13,9 & 0 & 0 & 0,629 \\
\hline Pensionado & 1 & 2,8 & 0 & 0 & \\
\hline Desempleado & 15 & 41,7 & 3 & 42,9 & \\
\hline Acceso a los servicios de salud & & & & & \\
\hline Sí & 17 & 47,2 & 4 & 57,1 & 0,631 \\
\hline No & 19 & 52,8 & 3 & 42,9 & \\
\hline Modo de contagio & & & & & \\
\hline Heterosexual & 9 & 25 & 7 & 100 & 0,001 \\
\hline Homosexual & 26 & 72,2 & 0 & 0 & \\
\hline Transfusión & 1 & 2,8 & 0 & 0 & \\
\hline Tratamiento antirretroviral en el & & & & & \\
\hline Sí & 34 & 94,4 & 7 & 100 & 0,523 \\
\hline No & 2 & 5,6 & 0 & 0 & \\
\hline Carga viral posterior a la pérdid & & & & & \\
\hline$<50$ copias & 4 & 11,1 & 0 & 0 & 0,354 \\
\hline$>50$ copias & 32 & 88,9 & 7 & 100 & \\
\hline Suspensión del tratamiento & & & & & \\
\hline Sí & 32 & 88,9 & 7 & 100 & 0,354 \\
\hline No & 4 & 11,1 & 0 & 0 & \\
\hline Falla del tratamiento & & & & & \\
\hline Sí & 16 & 94,1 & 1 & 100 & 0,354 \\
\hline No & 16 & 84,2 & 6 & 100 & \\
\hline Tiempo de evolución (años) & & & & & \\
\hline $0-4$ & 18 & 50 & 3 & 42,9 & 0,016 \\
\hline $5-9$ & 11 & 30,6 & 4 & 57,1 & \\
\hline $10-14$ & 4 & 11,1 & 0 & 0 & \\
\hline 15 o más & 3 & 8,3 & 0 & 0 & \\
\hline Tiempo de pérdida de seguimie & & & & & \\
\hline 3 & 8 & 22,2 & 0 & 0 & 0,368 \\
\hline 4 & 8 & 22,2 & 1 & 14,3 & \\
\hline 5 & 6 & 16,7 & 1 & 14,3 & \\
\hline 6 o más & 14 & 38,9 & 5 & 71,4 & \\
\hline Pérdida de seguimiento previo & & & & & \\
\hline Sí & 25 & 69,4 & 6 & 85,7 & 0,38 \\
\hline No & 11 & 30,6 & 1 & 14,3 & \\
\hline Tiempo de pérdida y falla del tr & & & & & \\
\hline 3 & 1 & 5,9 & 0 & 0 & 0,368 \\
\hline 4 & 3 & 17,6 & 0 & 0 & \\
\hline 5 & 4 & 23,5 & 0 & 0 & \\
\hline 6 o más & 9 & 52,9 & 1 & 100 & \\
\hline Tiempo de pérdida y hospitaliza & & & & & \\
\hline 3 & 1 & 33,3 & 0 & 0 & 0,584 \\
\hline 4 & 0 & 0 & 0 & 0 & \\
\hline 5 & 0 & 0 & 0 & 0 & \\
\hline 6 o más & 2 & 66,7 & 0 & 0 & \\
\hline Frecuencia de consumo alcoho & & & & & \\
\hline Nunca & 9 & 25 & 3 & 42,9 & 0,566 \\
\hline Una o menos veces al mes & 20 & 55,6 & 4 & 57,1 & \\
\hline 2 a 4 veces por mes & 6 & 16,7 & 0 & 0 & \\
\hline 2 a 3 veces por semana & 1 & 2,8 & 0 & 0 & \\
\hline Consumo de cigarrillo o tabaco & & & & & \\
\hline Sí & 9 & 25 & 1 & 14,3 & 0,539 \\
\hline No & 27 & 75 & 6 & 85,7 & \\
\hline Consumo de drogas & & & & & \\
\hline Sí & 3 & 8,3 & 0 & 0 & 0,009 \\
\hline No & 32 & 91,4 & 7 & 100 & \\
\hline Alteración del estado de ánimo & & & & & \\
\hline Sí & 20 & 55,6 & 7 & 100 & 0,026 \\
\hline No & 16 & 44,4 & 0 & 0 & \\
\hline Alteración del estado de ánimo & & & & & \\
\hline Sí & 6 & 30 & 3 & 43 & 0,016 \\
\hline No & 14 & 46,7 & 4 & 100 & \\
\hline
\end{tabular}

Nota: los valores en negrilla son estadísticamente significativos. 
Cuadro 2. Causas expuestas por los pacientes para explicar la pérdida de seguimiento

\begin{tabular}{lrr}
\hline Causas & $\mathbf{n}$ & $\%$ \\
\hline Factores administrativos del aseguramiento & & \\
No tenían trabajo & 14 & 32,55 \\
No tenían seguridad social & 13 & 30,23 \\
No tenían trabajo fijo & 2 & 4,65 \\
Trabas en el cambio de régimen de afiliación & 1 & 2,32 \\
Atraso en pagos de la empresa a la EPS & 1 & 2,32 \\
Problemas administrativos de la seguridad social & 3 & 6,97 \\
Suma del porcentaje & & 79,04 \\
Factores ocupacionales & & \\
Viajes & 4 & 9,3 \\
Carga laboral & 4 & 9,3 \\
Distancia al centro del programa de HIV & 2 & 4,65 \\
Estudios & 2 & 4,65 \\
Trabajo & 1 & 2,32 \\
Suma del porcentaje & & 30,22 \\
Factores psicosociales & & \\
Negación de la enfermedad & 2 & 4,65 \\
Falta de interés & 3 & 6,97 \\
Reacción negativa al tratamiento & 2 & 4,65 \\
Por voluntad propia & 1 & 2,32 \\
Búsqueda de medida alternativa & 1 & 2,32 \\
Abandono del tratamiento & 3 & 6,97 \\
Miedo a pedir permiso & 1 & 2,32 \\
Problema familiar & 1 & 2,32 \\
Depresión & 1 & 2,32 \\
Adicción al tramadol & 1 & 2,32 \\
Suma del porcentaje en factores psicosociales & & 37,16 \\
\hline & \multicolumn{2}{c}{. } \\
\hline
\end{tabular}

Nota: se establecieron 21 razones dadas por los 43 pacientes para ausentarse del programa de HIV/sida del Hospital Universitario San Ignacio. EPS: empresa promotora de salud

edad $(p=0,019)$, el no consumir alcohol $(p=0,028)$ y el desempleo $(p=0,001)$, resultaron asociadas estadísticamente con el riesgo de pertenecer al grupo de casos al hacer el ajuste según un modelo multivariado de regresión logística (cuadro 5). El ajuste del modelo de regresión logística se evaluó mediante el test de Hosmer y Lemeshow.

\section{Discusión}

El $20 \%$ de las personas que viven con la infección por HIV en los Estados Unidos no son conscientes de las implicaciones de su estado, y cerca de $50 \%$ de las diagnosticadas no están comprometidas con ningún programa de atención de pacientes con HIV. Cerca de $60 \%$ de los individuos infectados por el virus en ese país no reciben atención regular en los centros de cuidado de HIV. Del $40 \%$ restante, $80 \%$ requiere tratamiento antirretroviral y $80 \%$ de los individuos tratados tiene una carga viral indetectable ( $<50$ copias $/ \mathrm{ml})$.

Estos 210.000 individuos con carga viral indetectable corresponden a solo $19 \%$ de la población infectada con HIV en los Estados Unidos, en tanto que en $80 \%$ de los individuos infectados con el HIV la carga viral es detectable. Estas cifras posiblemente se explican por la diversidad de las variables que pueden afectar a dichos pacientes: diagnóstico tardío, inclusión irregular en programas de atención, fallas en el suministro del tratamiento antirretroviral y cumplimiento deficiente del tratamiento, barreras estas que impiden resultados óptimos en el tratamiento de los individuos con HIV (17).

El programa de HIV/sida del Hospital Universitario San Ignacio presentó una tasa de densidad de la incidencia de la pérdida en el seguimiento de 17,74 por 100 personas por año, mucho más alta que la observada en otros estudios $(3,72 ; 5,1$ y 9,1 por 100 personas por año) (18-20), aunque en otras poblaciones se han descrito tasas de pérdida en el seguimiento superiores a lo reportado en este estudio (25,1 y 21,4 por 100 personas por año) $(21,22)$. Las diferencias entre los estudios podrían deberse a las diversas metodologías aplicadas, así como a diferencias en la definición de pérdida en el seguimiento, en los tiempos de seguimiento de los estudios, en las poblaciones infectadas con HIV estudiadas y en las estructuras médicas locales $(18,20)$.

La probabilidad de retornar a un programa de atención de pacientes con HIV ha demostrado ser mayor en aquellos pacientes con empleo que en los desempleados (23). En otro estudio se observó la relación entre el desempleo, los bajos ingresos y la pérdida de seguimiento $(24,25)$. Clouse, et al., reportaron una asociación entre la pérdida en el seguimiento en jóvenes de 18 a 29 años y el desempleo (26), en tanto que Maskew, et al. (27), encontraron que las dificultades financieras de la población fueron la causa principal para no retomar los controles médicos; otras razones expuestas en este mismo estudio fueron sociales, y se relacionaban con la toma de los medicamentos y con factores ocupacionales como la carga laboral y la distancia del lugar de residencia al centro de atención del programa de HIV. Una de las razones por las que los pacientes considerados como perdidos en el seguimiento retornaron a los centros de atención, fue haber encontrado trabajo (28).

En otros estudios se ha reportado que la falta de seguridad social es un factor asociado a la pérdida de seguimiento $(29,30)$. Los resultados de este trabajo corroboran los reportes consultados en el sentido de que una situación de gran impacto 
Cuadro 3. Comparación entre los pacientes perdidos en el seguimiento y aquellos que lo continuaron (grupo de control)

\begin{tabular}{|c|c|c|c|c|c|}
\hline \multirow[t]{2}{*}{ Variables } & \multicolumn{2}{|c|}{ Grupo de control } & \multicolumn{2}{|c|}{$\begin{array}{l}\text { Pacientes perdidos en } \\
\text { el seguimiento }\end{array}$} & \multirow[t]{2}{*}{$p$} \\
\hline & $\mathbf{n}$ & $\%$ & $\mathbf{n}$ & $\%$ & \\
\hline \multicolumn{6}{|l|}{ Sexo } \\
\hline Masculino & 81 & 95,3 & 36 & 83,7 & \\
\hline Femenino & 4 & 4,7 & 7 & 16,3 & 0,027 \\
\hline \multicolumn{6}{|l|}{ Escolaridad } \\
\hline Ninguna & 1 & 1,2 & 0 & 0 & 0,657 \\
\hline Primaria incompleta & 2 & 2,4 & 2 & 4,7 & \\
\hline Primaria completa & 6 & 7,1 & 3 & 7 & \\
\hline Secundaria incompleta & 8 & 9,4 & 2 & 4,7 & \\
\hline Secundaria completa & 16 & 18,8 & 8 & 18,6 & \\
\hline Técnica & 26 & 30,6 & 14 & 32,6 & \\
\hline Universitaria & 21 & 24,7 & 14 & 32,6 & \\
\hline Postgrado & 5 & 5,9 & 0 & 0 & \\
\hline \multicolumn{6}{|l|}{ Modo de contagio } \\
\hline Heterosexual & 25 & 29,4 & 16 & 37,2 & \\
\hline Homosexual & 55 & 64,7 & 26 & 60,7 & 0,409 \\
\hline Transfusión & 1 & 1,2 & 1 & 2,3 & \\
\hline Bisexual & 4 & 4,7 & 0 & 0 & \\
\hline \multicolumn{6}{|l|}{ Tratamiento antirretroviral } \\
\hline Sí & 82 & 96,5 & 41 & 95,3 & 0,757 \\
\hline No & 3 & 3,5 & 2 & 4,7 & \\
\hline \multicolumn{6}{|l|}{ Tipo de ocupación } \\
\hline Obrero/empleado & 53 & 62,4 & 16 & 37,2 & \\
\hline Patrón/empleador & 3 & 3,5 & 3 & 7 & \\
\hline Trabajador familiar & 17 & 20 & 5 & 11,6 & \\
\hline no remunerado & 1 & 1,2 & 0 & 0 & \\
\hline Pensionado & 9 & 10,6 & 1 & 2,3 & \\
\hline No sabe & 2 & 2,4 & 0 & 0 & \\
\hline Desempleado & 0 & 0 & 18 & 41,9 & 0,000 \\
\hline \multicolumn{6}{|c|}{ Frecuencia de consumo de alcohol } \\
\hline Nunca & 50 & 58,8 & 12 & 27,9 & 0,006 \\
\hline Una o menos veces al mes & 29 & 34,1 & 24 & 55,8 & \\
\hline 2 a 4 veces al mes & 6 & 7,1 & 6 & 14 & \\
\hline 2 a 3 veces por semana & 0 & 0 & 1 & 2,3 & \\
\hline \multicolumn{6}{|l|}{ Consumo de cigarrillo o tabaco } \\
\hline Sí & 14 & 16,5 & 10 & 23,3 & 0,353 \\
\hline No & 71 & 83,5 & 33 & 76,7 & \\
\hline \multicolumn{6}{|l|}{ Consumo de drogas } \\
\hline Sí & 6 & 7,1 & 3 & 7,1 & 0,986 \\
\hline No & 79 & 92,9 & 39 & 92,9 & \\
\hline \multicolumn{6}{|c|}{ Alteración del estado de ánimo y atención psicológica } \\
\hline Sí & 13 & 100 & 9 & 100 & 0,349 \\
\hline No & 33 & 45,8 & 18 & 52,9 & \\
\hline \multicolumn{6}{|c|}{ Tiempo de evolución de la enfermedad en años } \\
\hline $0-4$ & 38 & 44,7 & 21 & 48,8 & 0,074 \\
\hline $5-9$ & 25 & 29,4 & 15 & 34,9 & \\
\hline $10-14$ & 21 & 24,7 & 4 & 9,3 & \\
\hline 15 o más & 1 & 1,2 & 3 & 7 & \\
\hline
\end{tabular}

Nota: los valores en negrilla son estadísticamente significativos

local es la pérdida del cubrimiento de los planes de aseguramiento en salud o los cambios que se presentan en dichos planes (paso del régimen contributivo al subsidiado); el factor más relevante reportado como razón para el abandono de los programas de atención es el paso del empleo formal a la condición de cesante.
Las mujeres con HIV se enfrentan a barreras similares en cuanto al proceso de aseguramiento en salud (31). Arrivillaga, et al., encontraron asociaciones estadísticamente significativas en los patrones cualitativos del cumplimiento del tratamiento y la posición social; las mujeres de posición social media y alta presentaron un mayor 
Cuadro 4. Análisis multivariado de las comparaciones que resultaron estadísticamente significativas, según se muestra en el cuadro 3

\begin{tabular}{|c|c|c|c|c|c|}
\hline \multirow[t]{2}{*}{ Variables } & \multicolumn{2}{|c|}{ Grupo de control } & \multicolumn{2}{|c|}{$\begin{array}{l}\text { Pacientes perdidos } \\
\text { en el seguimiento }\end{array}$} & \multirow[t]{2}{*}{$\mathbf{p}$} \\
\hline & $\mathbf{n}$ & $\%$ & $\mathbf{n}$ & $\%$ & \\
\hline \multicolumn{6}{|l|}{ Empleo } \\
\hline Con empleo o renta & 83 & 100,0 & 25 & 58,1 & 0,000 \\
\hline Desempleado & 0 & 0,0 & 18 & 41,9 & \\
\hline \multicolumn{6}{|l|}{ Consumo alcohol } \\
\hline Nunca & 50 & 58,8 & 12 & 27,9 & 0,004 \\
\hline Una o menos al mes & 29 & 34,1 & 24 & 55,8 & \\
\hline 2 o más veces al mes/semana & 6 & 7,1 & 7 & 16,3 & \\
\hline \multicolumn{6}{|c|}{ Tiempo de evolución de enfermedad en años } \\
\hline $0-9$ & 63 & 74,1 & 38 & 83,7 & 0,032 \\
\hline $10-14$ & 21 & 24,7 & 4 & 9,3 & \\
\hline 15 o más & 1 & 1,2 & 3 & 7 & \\
\hline \multicolumn{6}{|l|}{ Sexo } \\
\hline Masculino & 81 & 95,3 & 36 & 83,7 & 0,027 \\
\hline Femenino & 4 & 4,7 & 7 & 16,3 & \\
\hline Edad (promedio) & \multicolumn{2}{|c|}{40,3} & \multicolumn{2}{|c|}{34,0} & 0,000 \\
\hline
\end{tabular}

Nota: los valores en negrilla son estadísticamente significativos

Cuadro 5. Resultados del ajuste del modelo de regresión logística para la probabilidad de pérdida en el seguimiento de pacientes con HIV/sida

\begin{tabular}{|c|c|c|c|c|}
\hline \multirow[t]{2}{*}{$\begin{array}{l}\text { Variables en el } \\
\text { modelo }\end{array}$} & \multirow[t]{2}{*}{$\begin{array}{l}\text { Significación } \\
\text { estadística }\end{array}$} & \multirow[t]{2}{*}{$\begin{array}{c}\text { Razón de } \\
\text { momios }\end{array}$} & \multicolumn{2}{|c|}{$\begin{array}{l}\text { IC }_{95 \%} \text { para razón } \\
\text { de momios }\end{array}$} \\
\hline & & & Inferior & Superior \\
\hline Sexo & 0,017 & 7,787 & 1,903 & 31,863 \\
\hline Edad & 0,019 & 0,922 & 0,871 & 0,976 \\
\hline \multicolumn{5}{|l|}{ Tiempo de evolución } \\
\hline 0-9 años & 0,791 & 0,631 & 0,021 & 18,994 \\
\hline 10-14 años & 0,681 & 0,486 & 0,016 & 15,201 \\
\hline 15 o más años & 1,000 & & & \\
\hline \multicolumn{5}{|l|}{ Consumo de alcohol } \\
\hline Nunca consumió & 0,028 & 0,182 & 0,040 & 0,830 \\
\hline $\begin{array}{l}\text { Una vez o menos } \\
\text { al mes }\end{array}$ & 0,159 & 0,344 & 0,078 & 1,521 \\
\hline $\begin{array}{l}2 \text { o más veces al } \\
\text { mes/semana }\end{array}$ & 1,000 & & & \\
\hline Desempleo & 0,001 & 46,716 & 4,696 & 464,692 \\
\hline Constante & 0,588 & 3,643 & & \\
\hline
\end{tabular}

nivel de cumplimiento que aquellas de posición social baja. Además, se han descrito otras barreras críticas para el cumplimiento de los tratamientos, como el ser cuidadoras de niños con HIV y los estilos de afrontamiento individual (32).

Algunas características observadas en este estudio, como la reacción negativa al tratamiento, la depresión y la negación de la enfermedad, se han considerado como factores psicosociales asociados a la pérdida en el seguimiento en otros estudios (33). En este estudio, al ajustar el modelo multivariado de regresión logística, se encontró que las mujeres con una edad promedio de 34 años tenían riesgo de pertenecer al grupo de pacientes perdidos en el seguimiento. En su estudio, Rice, et al., reportaron que ser mujer y tener una edad entre los 15 y los 35 años, se asociaban con la pérdida en el seguimiento (34), lo cual contradice lo hallado en otros estudios, en los cuales la mayor pérdida en el seguimiento se presentó entre hombres jóvenes (35-37).

El consumo de alcohol se asoció a la pérdida de seguimiento en algunos estudios prospectivos llevados a cabo en Sudáfrica y Botsuana en torno a las características sociales y clínicas de pacientes con tratamiento antirretroviral, aunque dicho factor no predijo la pérdida en el seguimiento de los pacientes $(38,39)$.

En este estudio no se presentaron complicaciones clínicas en los 43 pacientes que se perdieron en el seguimiento clínico, aunque hubo tres casos de hombres en el grupo con más de seis meses de pérdida en el seguimiento que fueron hospitalizados por motivos que no tenían significación clínica relacionada con el tiempo de pérdida. Es posible que si el tiempo de seguimiento hubiese sido mayor, los eventos clínicos, e incluso la muerte, podrían haber sido más frecuentes, como lo demuestran algunos estudios en los que la pérdida de seguimiento se relacionó con la enfermedad avanzada y la muerte (40). Cabe señalar que estas situaciones no se producen inmediatamente después de la suspensión del tratamiento, sino que suelen ocurrir al cabo de un año y dependen, sobre todo, del recuento de células T CD4 en el momento en que se suspende el tratamiento. 
En el presente estudio se encontró que en 17 de los 39 (90,7\% del total) pacientes que suspendieron el tratamiento este falló después de la pérdida de seguimiento, lo cual se evidenció por dos cargas virales detectables después de reiniciarlo. La pérdida del seguimiento clínico, así como el incumplimiento del tratamiento, la inasistencia a las citas médicas y la supresión viral de corta duración, se consideran factores predictores del fracaso del tratamiento (41). Este es uno de los aspectos más preocupantes de la pérdida de seguimiento, dado que algunos pacientes suspenden completamente los medicamentos, mientras que otros los toman de manera irregular, con consecuencias complejas desde el punto de vista biológico, ya que esta es la principal causa de resistencia del HIV a los medicamentos.

Los alcances de estos hallazgos no solo tienen implicaciones clínicas (cambio del esquema de tratamiento antirretroviral con menores opciones en el futuro), sino también, implicaciones económicas (normalmente el segundo o tercer esquema es más costoso) y de salud pública (mayor riesgo de transmisión de virus resistentes). Este último aspecto es, quizá, el de mayor impacto en las políticas nacionales de salud y constituye el eje de las discusiones sobre las implicaciones para los pacientes, el sistema de salud y la población general, pues un individuo con infección por HIV que pierde el seguimiento $y$, como sucede muchas veces, suspende el tratamiento, representa un riesgo mayor de transmisión de virus resistentes a sus eventuales parejas.

Los principales obstáculos para lograr un resultado óptimo del tratamiento en personas con HIV/ sida, son el diagnóstico tardío, la demora en la vinculación a los centros de atención, la deficiente retención de los pacientes en dichos centros, la demora en el inicio del tratamiento antirretroviral y su incumplimiento (descontinuación o intermitencia del tratamiento), así como la ausencia de tratamiento para las enfermedades concomitantes no asociadas al HIV y las necesidades básicas insatisfechas (42-44).

La atención de los pacientes diagnosticados con HIV es crítico. En un estudio en Brasil, se establecieron las siguientes barreras para acceder a los servicios de atención de los pacientes con infección por HIV: los obstáculos relacionados con los servicios de salud (atención de mala calidad, prolongados tiempos de espera y problemas en el suministro de los medicamentos); los retos psicosociales y emocionales (miedo a la divulgación y la dificultad para aceptar el diagnóstico); los costos indirectos (transporte y ausentismo en el trabajo o la escuela); la percepción equivocada del riesgo y la toxicidad, y la complejidad de los esquemas de tratamiento antirretroviral. Los esfuerzos para eliminar las barreras más frecuentes pueden mejorar la continuidad asistencial a lo largo de las múltiples etapas de la atención (45).

El presente estudio es el primero en Colombia que ha establecido y analizado las causas de la pérdida de seguimiento en los pacientes que asisten a un programa de HIV. El estudio, sin embargo, tiene limitaciones metodológicas como la imposibilidad de contactar e identificar a todos los pacientes que podrían haber participado en el estudio, lo que disminuyó su poder, aunque ello no afectó de forma global la validez de las conclusiones. Algunas variables, como la de los pacientes fallecidos, la clasificación de las situaciones no reportadas y su significación clínica, y las posibles conductas del paciente durante la fase inicial de abandono del seguimiento (posiblemente asociadas a una falsa percepción del riesgo en salud), podrían haberse clasificado como casos. Otras variables que podrían haber sido de interés para el análisis incluyen el estrato social, el nivel de ingresos y el tipo de afiliación al régimen de salud. La selección de los casos puede haber comprometido la validez de los resultados, ya que no se pudo trabajar con el total de los pacientes, y el grupo con el cual se contó para el análisis no se seleccionó aleatoriamente.

Los resultados hallados para la población de pacientes con HIV del estudio no pueden extrapolarse a otras porque las causas de la pérdida de seguimiento pueden depender de la naturaleza de la población, el sitio de residencia, los métodos de seguimiento y la continuidad de los pacientes en los programas de atención.

Recientemente, se declaró constitucional la nueva ley de salud del país y se unificaron los beneficios de los regímenes contributivo y subsidiado, pero, en la realidad, cuando un paciente pierde su empleo no es trasladado al régimen subsidiado con la suficiente rapidez para garantizar la continuidad en sus tratamientos. Es necesario que el sistema de salud del país implemente un mecanismo para facilitar una transición ágil y rápida al régimen subsidiado en los casos de pérdida de empleo, y evitar la interrupción temporal o permanente de la atención integral a las personas con HIV. En todos los países se registran pérdidas considerables en 
el seguimiento, pero no se puede permitir que, además de las causas descritas en otros lugares, la pérdida del empleo se añada a las demás, sobre todo en un país como Colombia, con cifras elevadas de informalidad laboral.

Los hallazgos de este estudio sugieren que el ser mujer, joven, estar desempleado y consumir alcohol, se asocian a la pérdida en el seguimiento. Un mejor conocimiento de todos estos factores y su impacto en los programas de atención de HIV/sida, podría contribuir en la búsqueda de un mecanismo de prevención. La pérdida de seguimiento debe definirse para la población colombiana con HIV con la metodología adecuada y teniendo en cuenta el tiempo de seguimiento durante los estudios, el tipo de población y la región del país, para así diseñar estrategias que mejoren la retención de los pacientes y suministren información a largo plazo para evaluar los resultados de la atención a las personas con HIV.

El incumplimiento de los pacientes con HIV/sida debe analizarse teniendo en cuenta los factores relacionados con el tratamiento antirretroviral, así como los factores socioeconómicos, y los relacionados con la enfermedad y el sistema de salud, pues todos están implicados en la pérdida de seguimiento en los programas de atención de las personas que viven con HIV/sida.

\section{Conflicto de intereses}

Los autores de este trabajo no declaran ningún conflicto de intereses.

\section{Financiación}

No se recibió financiación externa para la elaboración de este trabajo.

\section{Referencias}

1. Antiretroviral Therapy Cohort Collaboration. Life expectancy of individuals on combination antiretroviral therapy in high-income countries: A collaborative analysis of 14 cohort studies. Lancet. 2008;372:293-9. http://dx.doi. org/10.1016/S0140-6736(08)61113-7

2. Smit C, Geskus R, Walker S, Sabin C, Coutinho R, Porter K, et al. Effective therapy has altered the spectrum of cause specific mortality following HIV seroconversion. AIDS. 2006;20:741-9. http://dx.doi.org/10.1097/01.aids. 0000216375.99560.a2

3. Kaplan JE, Hanson D, Dworkin MS, Frederick T, Bertolli $\mathrm{J}$, Lindegren $\mathrm{ML}$, et al. Epidemiology of human immunodeficiency virus associated opportunistic infections in the United States in the era of highly active antiretroviral therapy. Clin Infect Dis. 2000;30(Supl.1):S5-14. http://dx.doi. org/10.1086/313843
4. Grinspoon S, Carr A. Cardiovascular risk and bodyfat abnormalities in HIV-infected adults. N Engl J Med. 2005;352:48-62. http://dx.doi.org/10.1056/NEJMra041811

5. Núñez M. Hepatotoxicity of antiretrovirals: Incidence, mechanisms and management. J Hepatol. 2006;44:S132-9. http://dx.doi.org/10.1016/j.jhep.2005.11.027

6. Paterson DL, Swindells S, Mohr J, Brester M, Vergis EN, Squier C, et al. Adherence to protease inhibitor therapy and outcomes in patients with HIV infection. Ann Intern Med. 2000;133:21-30. http://dx.doi.org/10.7326/0003-4819-1331-200007040-00025

7. Low-Beer S, Yip B, O'Shaughnessy MV, Hogg RS, Montaner JS. Adherence to triple therapy and viral load response. J Acquir Immune Defic Synd. 2000;23:360-1.

8. Taiwo B. Understanding transmitted HIV resistance through the experience in the USA. Inter J Infect Dis. 2009;13:552-9. http://dx.doi.org/10.1016/j.jij.2008.10.008

9. Palella FJ, Baker RK, Moorman AC, Chmiel JS, Wood KC, Brooks JT, et al. Mortality in the highly active antiretroviral therapy era: Changing causes of death and disease in the HIV outpatient study. J Acquir Immune Defic Synd. 2006;43:27-34. http://dx.doi.org/10.1097/01. qai.0000233310.90484.16

10. D:A:D Study Group, Sabin CA, Worm SW, Weber $\mathbf{R}$, Reiss P, El-Sadr W, et al. Use of nucleoside reverse transcriptase inhibitors and risk of myocardial infarction in HIV-infected patients enrolled in the D:A:D study: A multicohort collaboration. Lancet. 2008;371:1417-26. http://dx. doi.org/10.1016/S0140-6736(08)60423-7

11. British HIV Association. Standards for HIV clinical care. London: BHIVA; 2007.

12. Ministerio de la Protección Social. Resolución número 3442 de 2006. Por la cual se adoptan las Guías de Práctica Clínica Basada en Evidencia para la prevención, diagnóstico y tratamiento de pacientes con $\mathrm{VIH} /$ sida y enfermedad renal crónica y las recomendaciones de los modelos de gestión programática en $\mathrm{VIH} /$ sida y de prevención y control de la enfermedad renal crónica. Bogotá: Ministerio de la Protección Social; 2006.

13. Ministerio de Protección Social, Fundación para la Investigación y Desarrollo de Salud y la Seguridad Social (FEDESALUD), Programa de Apoyo a la Reforma de Salud - PARS. Modelo de gestión programática en VIH/ sida. Bogotá: Ministerio de la Protección Social; 2006.

14. Mugavero MJ, Dávila JA, Nevin CR, Giordano TP. From access to engagement: Measuring retention in outpatient HIV clinical care. AIDS Patient Care STDS. 2010;24:60713. http://dx.doi.org/10.1089/apc.2010.0086

15. Weigel R, Hochgesang M, Brinkhof MW, Hosseinipour MC, Boxshall M, Mhango E, et al. Outcomes and associated risk factors of patients traced after being lost to follow-up from antiretroviral treatment in Lilongwe, Malawi. BMC Infect Dis. 2011;11:31. http://dx.doi.org/10.1186/14712334-11-31

16. Brinkhof MW, Pujades-Rodríguez M, Egger M. Mortality of patients lost to follow-up in antiretroviral treatment programmes in resource-limited settings: Systematic review and meta-analysis. PLoS One. 2009;4:e5790. http://dx.doi. org/10.1371/journal.pone.0005790 
17. Gardner EM, McLees MP, Steiner JF, Del Río C, Burman WJ. The spectrum of engagement in HIV care and its relevance to test-and-treat strategies for prevention of HIV infection. Clin Infect Dis. 2011;52:793-800. http://dx.doi. org/10.1093/cid/ciq243

18. Mocroft A, Kirk O, Aldins P, Chies A, Blaxhult A, Chentsova N, et al. Loss to follow-up in an international, multicentre observational study. HIV Med. 2008;9:261-9. http://dx.doi.org/10.1111/j.1468-1293.2008.00557.x

19. Krishnan S, Wu K, Smurzynski M, Bosch RJ, Benson CA, Collier AC, et al. Incidence rate of and factors associated with loss to follow-up in a longitudinal cohort of antiretroviraltreated HIV-infected persons: An AIDS Clinical Trials Group (ACTG) Longitudinal Linked Randomized Trials (ALLRT) analysis. HIV Clin Trials. 2011;12:190-200. http://dx.doi. org/10.1310/HCT1204-190

20. Elenga N, Georger-Sow MT, Messiaen T, Lamaury I, Favre I, Mathieu Nacher, et al. Incidence and risk factors for follow-up interruption of HIV infected patients in Guadeloupe. J Aids Clin Res. 2013:4:238. http://dx.doi. org/10.4172/2155-6113.1000238

21. Ochieng-Ooko V, Ochieng D, Sidle JE, Holdsworth M, Wools-Kaloustian K, Siika AM, et al. Influence of gender on loss to follow-up in a large HIV treatment programme in Western Kenya. Bull World Health Organ. 2010;88:681-8. http://dx.doi.org/10.2471/BLT.09.064329

22. Zhou J, Tanuma J, Chaiwarith R, Lee CK, Law MG, Kumarasamy $\mathbf{N}$, et al. Loss to follow-up in HIV-infected patients from Asia-Pacific Region: Result from TAHOD. AIDS Res Treat. 2012;2012:375217. http://dx.doi.org/10. $1155 / 2012 / 375217$

23. Larson BA, Brennan A, McNamara L, Long L, Rosen S, Sanne I, et al. Early loss to follow up after enrolment in preART care at a large public clinic in Johannesburg, South Africa. Trop Med Int Health. 2010;15(Supl.1):43-7. http:// dx.doi.org/10.1111/j.1365-3156.2010.02511.x.

24. Namusobya J, Semitala FC, Amanyire G, Kabami J, Chamie G, Bogere J, et al. High retention in care among HIV-infected patients entering care with CD4 levels $>350$ cells $/ \mu \mathrm{L}$ under routine program conditions in Uganda. Clin Infect Dis. 2013;57:1343-50. http://dx.doi.org/10.1093/cid/ cit490

25. Bassett IV, Wang B, Chetty S, Mazibuko M, Bearnot B, Giddy $\mathbf{J}$, et al. Loss to care and death before antiretroviral therapy in Durban, South Africa. J Acquir Immune Defic Syndr. 2009;51:135-9. http://dx.doi.org/10.1097/QAl.0b013e 3181a44ef2

26. Clouse K, Pettifor A, Maskew M, Bassett J, van Rie A, Gay $\mathbf{C}$, et al. Initiating antiretroviral therapy when presenting with higher CD4 cell counts results in reduced loss to followup in a resource-limited setting. AIDS. 2013;27:645-50. http://dx.doi.org/10.1097/QAD.0b013e32835c12f9

27. Maskew M, MacPhail P, Menezes C, Rubel D. Lost to follow up: Contributing factors and challenges in South African patients on antiretroviral therapy. S Afr Med J. 2007;97:853-7.

28. Gill VC, Krentz HB. Patient perspectives on leaving, disengaging, and returning to HIV care. AIDS Patient Care STDS. 2015;29:400-7. http://dx.doi.org/10.1089/apc. 2015.0001
29. Lebouché B, Yazdanpanah Y, Gérard Y, Sissoko D, Ajana F, Alcaraz I, et al. Incidence rate and risk factors for loss to follow-up in a French clinical cohort of HIV-infected patients from January 1985 to January 1998. HIV Med. 2006;7:140-5. http://dx.doi.org/10.1111/j.1468-1293.2006.00357.x

30. Hassan AS, Fielding KL, Thuo NM, Nabwera HM, Sanders EJ, Berkley JA. Early loss to follow-up of recently diagnosed HIV-infected adults from routine pre-ART care in a rural district hospital in Kenya: A cohort study. Trop Med Int Health. 2012;17:82-93. http://dx.doi.org/10.1111/j.13653156.2011.02889.x

31. Arrivillaga M. Análisis de las barreras para la adherencia terapéutica en mujeres colombianas con $\mathrm{VIH} /$ sida: cuestión de derechos de salud. Salud Pública Mex. 2010;52:350-6.

32. Arrivillaga M, Ross M, Useche B, Alzate ML, Correa D. Social position, gender role, and treatment adherence Colombian women living with HIV/AIDS: Social determinants of health approach. Rev Panam Salud Pública. 2009;26:50210. http://dx.doi.org/10.1590/S1020-49892009001200005

33. Udeagu CC, Webster TR, Bocour A, Michel P, Shepard CW. Lost or just not following up: Public health effort to re-engage HIV-infected persons lost to follow-up into HIV medical care. AIDS. 2013;27:2271-9. http://dx.doi. org/10.1097/QAD.0b013e328362fdde

34. Rice BD, Delpech VC, Chadborn TR, Elford J. Loss to follow-up among adults attending human immunodeficiency virus services in England, Wales, and Northern Ireland. Sex Transm Dis. 2011;38:685-90. http://dx.doi.org/10.1097/ OLQ.0b013e318214b92e.

35. Odafe S, Idoko O, Badru T, Aiyenigba B, Suzuki C, Khamofu H, et al. Patients' demographic and clinical characteristics and level of care associated with lost to follow-up and mortality in adult patients on first-line ART in Nigerian hospitals. J Int AIDS Soc. 2012;15:17424. http:// dx.doi.org/10.7448/IAS.15.2.17424.

36. Ndiaye B, Ould-Kaci K, Salleron J, Bataille P, Bonnevie $\mathbf{F}$, Choisy $\mathbf{P}$, et al. Incidence rate and risk factors for loss to follow-up in HIV-infected patients from five French clinical centres in Northern France - January 1997 to December 2006. Antivir Ther. 2009;14:567-75.

37. Nacher M, El Guedj M, Vaz T, Nasser V, Randrianjohany A, Álvarez $\mathrm{F}$, et al. Risk factors for follow-up interruption of HIV patients in French Guiana. Am J Trop Med Hyg. 2006;74:915-7.

38. Peltzer K, Ramlagan S, Khan MS, Gaede B. The social and clinical characteristics of patients on antiretroviral therapy who are 'lost to follow-up' in KwaZulu-Natal, South Africa: A prospective study. SAHARA J. 2011;8:179-86. http://dx.doi. org/10.1080/17290376.2011.9725002

39. Gust DA, Mosimaneotsile B, Mathebula U, Chingapane B, Gaul Z, Pals SL, et al. Risk factors for non-adherence and loss to follow-up in a three-year clinical trial in Botswana. PLoS One. 2011;6:e18435. http://dx.doi.org/10.1371/journal. pone.0018435.

40. Karcher H, Omondi A, Odera J, Kunz A, Harms G. Risk factors for treatment denial and loss to follow-up in an antiretroviral treatment cohort in Kenya. Trop Med Int Health. 2007;12:687-94. http://dx.doi.org/10.1111/j.13653156.2007.01830.x 
41. Robbins GK, Daniels B, Zheng H, Chueh H, Meigs JB, Freedberg KA. Predictors of antiretroviral treatment failure in an urban HIV clinic. J Acquir Immune Defic Syndr. 2007;44:30-7. http://dx.doi.org/10.1097/01.qai.0000248351. 10383.b7

42. Castilla J, Sobrino P, De La Fuente L, Noguer I, Guerra L, Parras F. Late diagnosis of HIV infection in the era of highly active antiretroviral therapy: Consequences for AIDS incidence. AIDS. 2002;16:1945-51.

43. Gardner LI, Metsch LR, Anderson-Mahoney P, Loughlin AM, del Río C, Strathdee S, et al. Efficacy of a brief case management intervention to link recently diagnosed HIVinfected persons to care. AIDS. 2005;19:423-31.

44. Lo W, MacGovern T, Bradford J. Association of ancillary services with primary care utilization and retention for patients with HIV/AIDS. AIDS Care. 2002;14(Supl.1):S4557. http://dx.doi.org/10.1080/0954012022014992049984

45. Hoffmann M, MacCarthy S, Batson A, Crawford-Roberts A, Rasanathan J, Nunn A, et al. Barriers along the care cascade of HIV-infected men in a large urban center of Brazil. AIDS Care. 2015;20:1-6. http://dx.doi.org/10.1080/0 9540121.2015 .1062462 